\title{
Ligand-based drug design of novel aldose reductase inhibitors
}

\author{
Magdaléna Májeková*,1 \\ 'Department of Biochemical Pharmacology, Center of Experimental Medicine SAS, Institute of Experimental Pharmacology \& \\ Toxicology, Slovak Academy of Sciences, Bratislava, Slovakia \\ *Author for correspondence: Tel.: +421 232295 709; Fax: +421 254775 928; magdalena.majekova@savba.sk
}

"Acidic conditions induce protonation of two important parts of the active site of the enzymatic complex: His110 (which often interacts with inhibitors via H-bond) and NADP ${ }^{+}$. As the resulting effect, the cavity of the active site is becoming more positive, when compared with the surrounding protein surface and physiological $\mathrm{pH}$."

First draft submitted: 19 April 2018; Accepted for publication: 20 April 2018; Published online: 30 November 2018

Keywords: aldose reductase inhibitors • diabetes mellitus Type $2 \bullet$ electrostatics $\bullet$ ligand-based drug design

\section{Current state of design of aldose reductase inhibitors}

Proportionally to the increasing number of patients with diabetes mellitus, the late diabetic complications cause serious health problems worldwide. Aldose reductase (AR) is as a rate-limiting enzyme in the polyol pathway, one of the main mechanisms of glucose toxicity. Attempts to find efficient and safe inhibitors of aldose reductase (ARIs) are dated early after the very identification of AR [1]. However, from the group of AR inhibitors, there is only one compound used clinically (epalrestat). A further candidate, fidarestat, is expected to appear among US FDA approved drugs in the near future.

Compounds found and proven in preclinical research as prospective drugs failed in clinical trials either for excessive adverse effects or due to a weak bioavailability. For this reason, the strategies in search of new ARIs should be reconsidered in order to identify the strong and poor sides of each drug design methodology in this field. This editorial is to point out the specific potential characteristic for ligand-based drug design of ARIs.

\section{Specific features of AR}

Aldose reductase (EC: 1.1.1.21) belongs to the aldo-keto reductase superfamily [2]. The enzyme is important for many metabolic processes, but its main role in the framework of the polyol pathway is to catalyze the NADPHdependent conversion of glucose to sorbitol with simultaneous change of NADPH to $\mathrm{NADP}^{+}$. Under pathologic conditions, this leads to accumulation of sorbitol in cells and destruction of tissues. The 3D structure of AR has been explored in many research works. Besides, numerous x-ray crystallographic works, also a neutron diffraction experiment was documented [3]. The catalytic mechanism was discussed in detail by Blakeley et al. [4].

AR catalyzes many substrates, as lipid aldehydes, methyl glyoxal, aliphatic and aromatic aldehydes, daunorubicin, doxorubicin among others. [5]. With the exception of PGH2 transformation to PGD2, which proceeds in the absence of cofactors NADPH or $\mathrm{NADP}^{+}$, all other known catalytic processes of AR are NADPH-dependent. Participation of the cofactor NADPH means that the whole catalysis is a two-substrate process (sequentially ordered bi-bi process), a fact not often discussed in the evaluation of AR inhibition experiments [6]. In the first step of the process of aldehyde reduction, for example, glucose to alcohol (e.g., sorbitol), the enzyme binds with NADPH (the first substrate), which induces the conformational changes to create a binary complex E*•NADPH. Only this complex is able to bind aldehyde (the second substrate), which also provokes the formation of a new (yet ternary) complex $E^{*} \bullet$ NADPH•aldehyde, readily changed via multistep reaction [4] and consumption of one proton to $\mathrm{E}^{*} \bullet \mathrm{NADP}^{+} \bullet$ alcohol complex (i.e., complex of the enzyme with both products). Leaving the second substrate, the enzyme is in the state $\mathrm{E}^{*} \bullet \mathrm{NADP}^{+}$, which is known as an object for binding of most AR inhibitors. Without binding of an inhibitor, $\mathrm{NADP}^{+}$is released and the enzyme relaxes to the initial state. 
In the framework of this process, there are only two steps when inhibition could be achieved by an external compound:

- Before NADPH binding and subsequent fixation of the 'safety-belt', in other words, pushing the loop of residues Gly213-Leu226 to [7];

- After leaving the second product, in other words, by attacking the complex $\mathrm{E}^{*} \bullet \mathrm{NADP}^{+}$.

Structurally, almost all inhibitors known so far were identified in complex with $\mathrm{E}^{*} \bullet \mathrm{NADP}^{+}{ }_{[8,9]}$. The glutathione analog S-(1,2-dicarboxyethyl) glutathione (DCEG) was found to be a competitive inhibitor of AR with glutathionepropanal as substrate [10]. The complex of AR with DCEG and NADPH provided the only ternary structure of the complex of AR, cofactor and inhibitor with $\mathrm{NADPH}$ and not $\mathrm{NADP}^{+}$(pdb 2f2k, [11]). In contrast to the product-like ligands, as lidorestat or fidarestat, DCEG represents substrate-like inhibitors.

Further determining property of AR, which is important for ligand-based drug design, is a dependence of $A R$ catalytic activity on $\mathrm{pH}$. The optimum value of $\mathrm{pH}$ for the catalysis of aldehydes as glucose or glyceraldehyde is 6.2; however, the reduction of PGH2 to PGD2 (in the absence of $\mathrm{NADPH}$ ) requires the optimum value of $\mathrm{pH}=$ 8.5, while the same substrate is reduced to PGF2 in the presence of NADPH optimally at $\mathrm{pH}=5.0$ [12]. Acidic conditions induce protonation of two important parts of the active site of the enzymatic complex: His110 (which often interacts with inhibitors via $\mathrm{H}$-bond) and $\mathrm{NADP}^{+}$. As the resulting effect, the cavity of the active site is becoming more positive, when compared with the surrounding protein surface and physiological $\mathrm{pH}$.

Electrostatics of the active site works as a driving force for attracting the negatively charged acetic groups of such inhibitors as zopolrestat, epalrestat or lidorestat, which are inhibitors derived from the first known compounds capable to inhibit AR - organic acids [1]. Electrostatic interaction, mainly one of the first orders (charge-field interaction) belongs to the long-range interactions. As such, it is proper to drive a molecule from the larger distance, but alone it is not so good for stabilization of the molecule in the active site, as the energetic minimum of this interaction is broader than that of others (e.g., H-bond interaction). In the situation when inhibition means to prevent release of the co-factor from the protein, this interaction is not sufficient. Inhibitors with acetic functional group are therefore the best example of ligand-based design of ARIs. Coming from organic acids, researchers proposed structures which provided more and more interactions with residues of the active site. Besides, the hydrophobic interaction between methyl of acetyl group and nicotinamide ring, which is typical for all inhibitors of this type, we can observe an increasing number of H-bonds when inspecting inhibitors from weak to strong (alrestatin, epalrestat, tolrestat, zenarestat, cemtirestat, zopolrestat, lidorestat; corresponding pdb structures 1az1, 4jir, 2fzd, 1iei, 4qx4, 2fz8, 1z3n). The tricyclic framework, combining the skeleton rigidity with the proper placement of the functional acetyl group, was found to be advantageous for exact setting of new substituents which could ensure other properties, for example, high selectivity toward aldehyde reductase [13].

\section{Hydantoin derivatives}

Sorbinil, the first representative of hydantoin AR inhibitors, was designed without exact knowledge of the ALR2 binding site, only as an attempt to mimic the substrate glucose or the co-factor NADPH. Although, the clinical trials did not confirm the in vivo activity expected, it was later often used for molecular modeling studies and gave rise, on the basis of ligand-based design, to the most prospective hydantoin inhibitor - fidarestat [14].

\section{Inhibitors of natural origin}

Natural compounds have a long tradition in the treatment of diabetes, mainly in the traditional Chinese medical system, which has categorized diabetes as a separate disease [15]. Recent studies, re-established with means of modern experimental methods, found a large number of natural compounds able to affect chronic diabetic complications. Many of them are able to inhibit AR. The 4-hydroxycoumarin, 4,2',4'-trihydroxychalcones, tetra- and penta-Ogalloyl-glucosides, as well as ellagic acid and its derivatives proved to be the most efficient inhibitors with $\mathrm{IC}_{50}$ achieving the value $0.02 \mu \mathrm{m} / 1$ [16]. On taking polyphenolic compounds as leads for ligand-based design of ARIs, a harder control is required due to the greater risk for pan-assay interference compounds (PAINS) character.

\section{Future perspective}

In order to find an efficient and safe drug from the group of ARIs means, besides constructing a powerful inhibitor, to avoid the reasons of clinical trial failures, in other words, adverse effects and a low bioavailability. To fulfill the 
first condition, a high selectivity toward aldehyde reductase should be achieved, which is connected with interaction of a ligand and residues of the so-called specificity pocket, mainly with Leu300, Phe122 and Trp219. Creating an H-bond with Leu300 seems to be the best guarantee of high selectivity, as was observed also for fidarestat [17]. Opening the specificity pocket is often, yet not always, connected with selectivity [18].

Carboxylic acid derivatives wearing a negatively charged group are often handicapped for their low bioavailability. However, the real transport through membrane compartments is given by the total permeability coefficient, not only by partitioning, as in each hydrophilic compartment the almost same acid-basic balance is established. Indole compounds, even when wearing an acetyl substituent, have reasonable values of the permeability coefficient [19].

The last point to be emphasized is the necessity of joining the ARI activity with another type of molecular mechanism, creating thus the compound with polypharmacological effect. Diabetes is a multifactorial disease and therefore needs a multi-target approach in designing new drugs. For this aim, the ligand-based approach is a good choice.

\section{Financial and competing interests disclosure}

This work was supported by the grants of the Slovak Research and Development Agency under the contract No. APVV-15-0455 and the Scientific Grant Agency VEGA grant 2/0127/18. The authors have no other relevant affiliations or financial involvement with any organization or entity with a financial interest in or financial conflict with the subject matter or materials discussed in the manuscript apart from those disclosed.

No writing assistance was utilized in the production of this manuscript.

\section{References}

1. Hayman S, Kinoshita JH. Isolation and properties of lens aldose reductase. J. Biol. Chem. 240, 877-882 (1965).

2. Mindnich RD, Penning TM. Aldo-keto reductase (AKR) superfamily: genomics and annotation. Hum. Genomics 3, 362-370 (2009).

3. Hazemann I, Dauvergne MT, Blakeley MP et al. High-resolution neutron protein crystallography with radically small crystal volumes: application of perdeuteration to human aldose reductase. Acta Crystallogr. D Biol. Crystallogr. 61(Pt 10), 1413-1417 (2005).

4. Blakeley MP, Ruiz F, Cachau R et al. Quantum model of catalysis based on a mobile proton revealed by subatomic x-ray and neutron diffraction studies of h-aldose reductase. PNAS 105, 1844-1848 (2008).

5. Oppermann U. Complex relationships of mammalian carbonyl- and quinone-reducing enzymes and their role in physiology. Annu. Rev. Pharmacol. Toxicol. 47, 293-322 (2007).

6. Petrash JM. All in the family: aldose reductase and closely related aldo-keto reductases. Cell. Mol. Life Sci. 61, 737-749 (2004).

7. Biadene M, Hazemann I, Cousido A et al. The atomic resolution structure of human aldose reductase reveals that rearrangement of a bound ligand allows the opening of the safety-belt loop. Acta Crystallogr. D Biol. Crystallogr. 63(Pt 6), 665-672 (2007).

8. Klebe G, Krämer O, Sotriffer C. Strategies for the design of inhibitors of aldose reductase, an enzyme showing pronounced induced-fit adaptations. Cell. Mol. Life Sci. 61, 783-793 (2004).

9. Maccari R, Ottanà R. Targeting aldose reductase for the treatment of diabetes complications and inflammatory diseases: new insights and future directions. J. Med. Chem. 58, 2047-2067 (2015).

10. Dixit BL, Balendiran GK, Watowich SJ et al. Kinetic and structural characterization of the glutathione-binding site of aldose reductase. J. Biol. Chem. 275, 21587-21595 (2000).

11. Singh R, White MA, Ramana KV et al. Structure of a glutathione conjugate bound to the active site of aldose reductase. Proteins 6 , 101-110 (2006).

12. Nagata N, Kusakari Y, Fukunishi Y et al. Catalytic mechanism of the primary human prostaglandin F2a synthase, aldo-keto reductase $1 \mathrm{~B} 1$ - prostaglandin D2 synthase activity in the absence of NADP(H). FEBS J. 278, 1288-1298 (2011).

13. Majekova M, Ballekova J, Prnova M et al. Structure optimization of tetrahydropyridoindole-based aldose reductase inhibitors improved their efficacy and selectivity. Bioorg. Med. Chem. 25, 6353-6360 (2017).

14. Oka M, Matsumoto Y, Sugiyama $S$ et al. A potent aldose reductase inhibitor, (2S,4S)-6-fluoro-2', 5'-dioxospiro[chroman-4,4'-imidazolidine]-2-carboxamide (Fidarestat): its absolute configuration and interactions with the aldose reductase by x-ray crystallography. J. Med. Chem. 43, 2479-2483 (2000).

15. Li WL, Zheng HC, Bukuru J et al. Natural medicines used in the traditional Chinese medical system for therapy of diabetes mellitus. J. Ethnopharmacol. 92, 1-21 (2004).

16. Kawanishi K, Ueda H, Moriyasu M. Aldose reductase inhibitors from the nature. Curr. Med. Chem. 10, 1353-1374 (2003).

17. El-Kabbani O, Carbone V, Darmanin C et al. Structure of aldehyde reductase holoenzyme in complex with the potent aldose reductase inhibitor fidarestat: implications for inhibitor binding and selectivity. J. Med. Chem. 48, 5536-5542 (2005). 
18. Stefek M, Soltesova Prnova M, Majekova M et al. Identification of novel aldose reductase inhibitors based on carboxymethylated mercaptotriazinoindole scaffold. J. Med. Chem. 58, 2649-2657 (2015).

19. Bean RC, Shepherd WC, Chan H. Permeability of lipid bilayer membranes to organic solutes. J. Gen. Phys. 52, 495-508 (1968). 